\title{
Risk of Hypocalcemia after Total Thyroidectomy and Bilateral Central Neck Dissection in Patients with Well Differentiated Thyroid Carcinoma
}

\author{
Muhammad Gaber Gohar ${ }^{1, *}$ MB BCh, Ahmad El-Sayed Mohamed ${ }^{2}$ MD \& Hamed Ahmed Al-Azzouny ${ }^{2}$ MD
}

* Corresponding Author:

Muhammad Gaber Gohar mgohar0712@gmail.com

Received for publication October 1, 2020; Accepted January 1, 2021; Published online January 1, 2021.

Copyright 2020 The Authors published by Al-Azhar University, Faculty of Medicine, Cairo, Egypt. All rights reserved. This an open-access article distributed under the legal terms, where it is permissible to download and share the work provided it is properly cited. The work cannot be changed in any way or used commercially.

doi: $10.21608 /$ aimj.2021.44026.1327

${ }^{1}$ Surgical Oncology Department, Damanhour Oncology Center Albeheira, Egypt.

${ }^{2}$ Surgical Oncology Department, Faculty of Medicine, Al-Azhar University, Cairo, Egypt.

\begin{abstract}
Background: Hypocalcaemia is a grave complication after thyroid surgery with signs ranging from paresthesia up to laryngospasm. Avoiding this complication is mandatory to avoid lifelong calcium and vitamin D supplements.

Aim of work: To assess the risk of hypocalcaemia after thyroid surgery and the factors contributing to it.

Patient and methods: This study was conducted on 50 patients with well differentiated thyroid carcinoma admitted to Sayed Galal hospital. They underwent total thyroidectomy and central neck dissection and assessed for postoperative hypocalcaemia by measuring serum calcium and parathormone levels, then they were divided into 3 groups: A, B and C.

Results: Group B patients had transient hypocalcaemia and PTH levels were in normal range, thus suffering from parathyroid dysfunction. Calcium levels returned to normal range after a mean of 15.5 days. Group C patients remained hypocalcemic for over six months and PTH levels were below normal range thus defining absolute hypoparathyroidism.

In terms of age, gender, tumor size and stage, group C patients were older, had no female prevalence, had slightly larger tumors and presented at late stages. Preoperative calcium levels were not a risk factor as group $\mathrm{C}$ patients had the highest mean of ionized calcium and group B patients had lower mean of total calcium level than those of group C.

Conclusion: The most important risk factor for post-thyroidectomy hypocalcaemia is the surgical technique. Meticulous dissection to preserve the blood supply to the parathyroid glands and careful handling of the parathyroid tissue are equally important to preserving the parathyroids themselves. Other risk factors include older age, large tumors and late stages.
\end{abstract}

Keywords: Hypocalcaemia; Thyroid Carcinoma; Total Thyroidectomy; Central Neck Dissection; Hypoparathyroidism.

Disclosure: The authors have no financial interest to declare in relation to the content of this article. The Article Processing Charge was paid for by the authors.

Authorship: All authors have a substantial contribution to the article.

\section{INTRODUCTION}

Thyroid carcinoma is the $5^{\text {th }}$ most common cancer in Egyptian women. ${ }^{1}$ It has many pathological types but the most common variant is the papillary variant representing about $85 \%$ of cases. $^{2}$ Papillary carcinoma itself has many histopathologic subtypes. ${ }^{3}$ Malignant cells disseminate through lymphatics, that run adjacent to the gland's venous drainage, to the draining lymph nodes in level VI of the neck. Adjacent to the thyroid gland lie the parathyroid glands that regulate serum calcium level by secreting the parathyroid hormone. ${ }^{5}$

In well differentiated thyroid carcinomas, surgery is an important step in the treatment approach. ${ }^{6}$
Postoperative hypocalcaemia may develop as a complication of surgery and it may be transient or permanent. ${ }^{7}$ Symptoms of hypocalcemia range from numbness to tetany and laryngeal spasm. ${ }^{8}$ This occurs as a result of trauma to the parathyroid glands or ligation of their blood supply during surgery. ${ }^{9}$ Measuring parathyroid hormone (PTH) level can early predict postoperative hypoparathyroidism. ${ }^{10}$

\section{PATIENT AND METHODS}

This study was conducted on fifty patients with well differentiated thyroid carcinoma and cervical lymphadenopathy admitted to Sayed Galal Hospital in the period from June 2018 to October 2019. Patients were subjected to total thyroidectomy and bilateral central neck dissection followed by assessment of hypocalcaemia over a period of six 
months by measuring postoperative total and ionized calcium levels in all patients and measuring PTH level in hypocalcemic patients.

Diagnosis of patients was based on a combination of detailed history, thorough clinical examination, neck ultrasonography, neck computed tomography (CT) in advanced cases and pathological examination through fine needle aspiration cytology (FNAC).

Exclusion criteria included history of previous neck surgery, history of hypoparathyroidism, patients on Iodine therapy and patients with disorders of calcium metabolism (e.g.; chronic renal disease, sarcoidosis). Detailed history was investigated for all patients and data was collected regarding age, gender, medical and drug history. Complete physical examination was done including voice assessment and indirect laryngoscopy. Laboratory investigations were done preoperatively including free $\mathrm{T}_{3}$, free $\mathrm{T}_{4}, \mathrm{TSH}$, serum total and ionized calcium. Neck ultrasound was done for all patients and CT was conserved for those with advanced tumors. FNAC was done in all patients for a pathological diagnosis and only cases with well differentiated carcinomas were included. Patients included in this study were subjected to extracapsular total thyroidectomy with bilateral central node dissection (level VI node dissection on both sides) with great attention to preserving all the parathyroid glands and their blood supply by dividing the branches of the inferior thyroid arteries supplying the thyroid gland and sparing the main inferior thyroid arteries and their branches to the parathyroid glands. Postoperative total and ionized calcium levels were measured on the first postoperative day. In hypocalcemic cases, PTH level was measured and closer monitoring of serum calcium levels was done. All hypocalcemic patients were started on calcium and vitamin D supplements with trials to wean them off going hand in hand with measuring serum calcium levels.

Follow up visits were scheduled for all patients after a month, 2 months and 6 months. With each visit thyroid hormone profile and voice assessment were done and serum total and ionized calcium were measured. Patients were divided into three groups: group A normocalcemic, group B hypocalcemic euparathyroid and group $\mathrm{C}$ hypocalcemic hypoparathyroid.

In this study, postoperative total and ionized calcium levels were used to screen for postoperative hypocalcemia before developing signs and symptoms of hypocalcemia. PTH levels were measured for cases with proved hypocalcemia to assess the function of the parathyroid glands and predict cases with permanent hypocalcemia. ${ }^{11}$ Analysis of clinicopathologic data and comparing values in different groups helps identify risk factors for permanent hypocalcemia.

Data was analyzed using Microsoft Excel and SPSS software for Windows (version 1.0.0.137; SPSS Inc., Chicago, IL). The continuous variables were presented as $\mathrm{x}$ mean + Standard Deviation (to measure the central tendency of data and the distribution of data around their mean value).

\section{RESULTS}

Fifty patients were recruited for this study and their clinicopathologic characteristics were investigated.

Of all patients, 38 were females (76\%) and only 12 were males $(24 \%)$. Twenty four patients were younger than 45 years old (48\%), while 26 were 45 years old or older $(52 \%)$. Forty six patients were diagnosed as papillary thyroid carcinoma (92\%), while the remaining 4 patients were diagnosed as follicular carcinoma $(8 \%)$. Tumors were of variable sizes with mean diameter of $19.96 \mathrm{~mm}$ as the largest diameter. Thirty four cases presented with single tumors $(68 \%)$ and the remaining 16 cases presented with multiple tumors (32\%). Two cases only presented with invasive tumors (4\%), 36 presented with focal invasion of the gland capsule (72\%) and the remaining 12 cases showed no capsular invasion (24\%). Thirty eight patients were staged as stage I thyroid carcinoma (76\%), 6 patients were staged as stage II $(12 \%)$ and 6 patients were staged as stage IVB (12\%). Table 1

Fourteen patients showed postoperative hypocalcaemia $(28 \%)$. PTH levels were measured for them and 4 of them showed hypoparathyroidism (8\%) while the remaining 10 patients had normal PTH levels (20\%). Accordingly, patients were divided into 3 groups; group $\mathrm{A}$ with patients who were normocalcemic, group B with 10 hypocalcemic patients with normal PTH levels and group C with 4 patients with hypoparathyroidism. Figure 1

In terms of age, gender prevalence and tumor size, both groups A and B had close values compared to group C. Group C patients were much older with no female prevalence and presented with larger tumors. Figure 2

Groups A and B presented mostly with early stage tumors while group $\mathrm{C}$ had tumors at later stages namely stages II and IVB. Group C patients had higher mean of preoperative serum ionized calcium compared to other groups. Figure 3

Group A had 2 cases of locally invasive tumors while both groups B and C had no invasive tumors. Group A had more cases with single tumors than with multiple tumors (2.6:1) while groups B and $\mathrm{C}$ had less ratios (1.5:1 and 1:1 respectively). Table 2 


\begin{tabular}{|c|c|c|}
\hline \multicolumn{2}{|c|}{ Characteristic } & Value (\%) \\
\hline \multirow{2}{*}{ Sex } & Male & $12(24)$ \\
\hline & Female & $38(76)$ \\
\hline \multirow{2}{*}{ Age } & $<45$ & $24(48)$ \\
\hline & $\geq 45$ & $26(52)$ \\
\hline \multirow{2}{*}{ Diagnosis } & Papillary carcinoma & $46(92)$ \\
\hline & Follicular carcinoma & $4(8)$ \\
\hline \multicolumn{2}{|c|}{ Mass size $(\mathrm{mm})$} & $19.96 \pm 9.5$ \\
\hline \multirow{2}{*}{$\begin{array}{l}\text { Multifoca } \\
\text { lity }\end{array}$} & Single & $34(68)$ \\
\hline & Multiple & $16(32)$ \\
\hline \multirow{3}{*}{$\begin{array}{l}\text { Capsular } \\
\text { invasion }\end{array}$} & Invasive & $2(4)$ \\
\hline & Focally invasive & $36(72)$ \\
\hline & Non invasive & $12(24)$ \\
\hline \multirow{5}{*}{ T stage } & $\mathrm{T} 1$ & $36(72)$ \\
\hline & $\mathrm{T} 2$ & $8(16)$ \\
\hline & T3a & $2(4)$ \\
\hline & $\mathrm{T} 3 \mathrm{~b}$ & $2(4)$ \\
\hline & $\mathrm{T} 4 \mathrm{a}$ & $2(4)$ \\
\hline \multirow{3}{*}{$\mathrm{N}$ stage } & NOa & $14(28)$ \\
\hline & N1a & $12(24)$ \\
\hline & N1b & $24(48)$ \\
\hline \multirow{2}{*}{ M stage } & M0 & $44(88)$ \\
\hline & M1 & $6(12)$ \\
\hline \multirow{3}{*}{$\begin{array}{l}\text { TNM } \\
\text { stage }\end{array}$} & $\mathrm{I}$ & $38(76)$ \\
\hline & II & $6(12)$ \\
\hline & IVB & $6(12)$ \\
\hline
\end{tabular}

Table 1: Clinicopathologic characteristics of all patients.

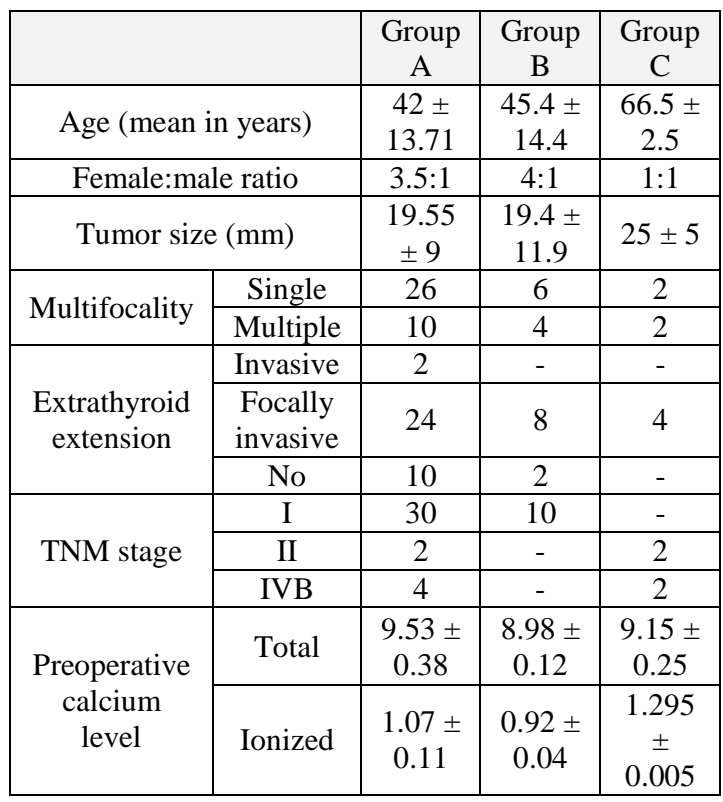

Table 2: Clinicopathologic characters of patient groups

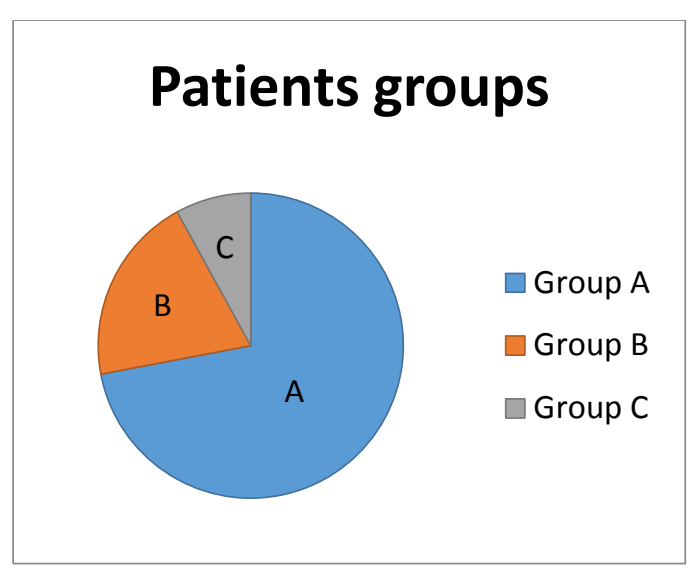

Fig. 1: Patient groups.

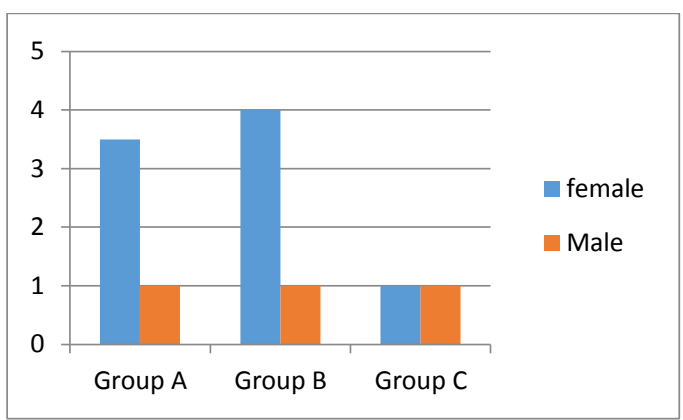

Fig. 2: Female to male prevalence in patient groups

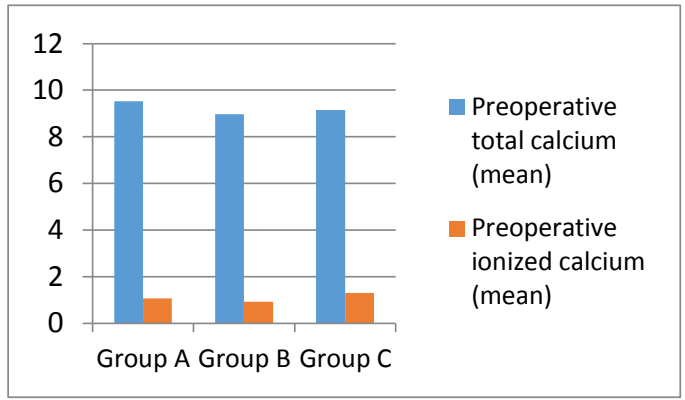

Fig. 3: Comparing means of preoperative total and ionized calcium levels in patient groups

\section{DISCUSSION}

Hypocalcaemia is one of the major complications of total thyroidectomy and central neck dissection due to the close anatomic relation of the thyroid gland and the parathyroid glands which regulate the blood calcium level through secretion of parathormone (PTH). ${ }^{12}$

Due to the short half-life of PTH, early postoperative measurement of PTH can help diagnose hypoparathyroidism before appearance of signs of hypocalcaemia. $^{13}$

Yet while hypoparathyroidism causes hypocalcaemia, postoperative hypocalcaemia may occur despite normal PTH level. ${ }^{14}$

In this study, assessment of hypocalcemia was done by measuring total and ionized calcium after surgery. 
Measurement of PTH was done in hypocalcemic cases.

In group A patients, both total and ionized calcium levels remained within normal range and they didn't show any clinical signs of hypocalcaemia. In group B patients there was an evident drop in both total and ionized calcium levels beyond normal range and patients' symptoms varied in severity from mild peripheral hypothesia to circumoral hypothesia and carpopedal tetany. PTH levels were measured for patients of this group and all were within normal range and mostly near the lower end of the normal range (median $=23 \mathrm{pg} \backslash \mathrm{ml}, \mathrm{sd}= \pm 4.32$ ). Some authors defined parathyroid insufficiency as having clinical signs and symptoms of hypocalcaemia while having normal PTH level. ${ }^{15}$ Patients of group C presented with frank signs of hypocalcemia, which was confirmed by decreased levels of both total and ionized calcium, and their PTH levels showed hypoparathyroidism.

Group C patients continued to receive oral calcium and vitamin D supplements for over 6 months. Authors suggested different time points to define permanent hypocalcaemia. Some authors suggested a 6 month period of calcium and vitamin D supplements before declaring permanent hypocalcaemia ${ }^{16}$ while others suggested a year. ${ }^{17}$

In all group B patients, total and ionized calcium levels returned to normal range and oral calcium and vitamin D supplements were discontinued within a mean of 15.57 days. This is consistent with known cases of post-thyroidectomy parathyroid dysfunction in which calcium levels return to normal range within a time window of a week up to a month after surgery. ${ }^{18}$

In terms of age, gender, tumor size and stage, group $\mathrm{C}$ patients were older, had no female prevalence, had slightly larger tumors and presented at later stages. Preoperative calcium level was not a risk factor as group $\mathrm{C}$ patients had the highest mean of ionized calcium and group B patients had lower mean of total calcium level than those of group C.

The results seem to demonstrate that the principal mechanism underlying postoperative hypocalcemia is parathyroid gland injury related to intraoperative trauma and that according to the extent of trauma, parathyroid dysfunction and resulting transient hypocalcaemia occur with mild trauma with intact blood supply to the parathyroid glands, while hypoparathyroidism and resulting permanent hypocalcaemia occur with severe trauma or inadvertent ligation of the blood supply to the parathyroid glands.

\section{CONCLUSION}

The most important risk factor for postthyroidectomy hypocalcaemia is the surgical technique. Meticulous dissection to preserve the blood supply to the parathyroid glands and careful handling of the parathyroid tissue are equally important to preserving the parathyroids themselves.
Other risk factors include older age, large tumors and late stages.

Limitations to this study included lack of preoperative measurement of $\mathrm{PTH}$, thus the change in perioperative PTH level couldn't be assessed. Serum albumin was not measured, so corrected calcium level wasn't calculated but there is an increasing tendency towards use of ionized calcium instead of corrected calcium for a more accurate calcium level. Serum magnesium level wasn't measured, while magnesium has a role in PTH release.

\section{REFERENCES}

1. Ibrahim, A. S., Khaled, H. M., Mikhail, N. N., et al. Cancer Incidence in Egypt: Results of the National Population-Based Cancer Registry Program. Journal of Cancer Epidemiology. 2014; $1-18$

2. Baloch, Z. W., \& LiVolsi, V. A. Special types of thyroid carcinoma. Histopathology. 2017; 72(1), 40-52.

3. Nath M.C. and Erickson L.A. Aggressive Variants of Papillary Thyroid Carcinoma: Hobnail, Tall Cell, Columnar, and Solid. Advances In Anatomic Pathology. 2018; 25(3): 172-9.

4. Li G, Lei J, Peng Q, et al. Lymph node metastasis characteristics of papillary thyroid carcinoma located in the isthmus: A single-center analysis. Medicine (Baltimore). 2017; 96(24): e7143.

5. Rudin AV, McKenzie TJ, Wermer RA, et al. Primary Hyperparathyroidism: Redefining Cure. Am Surg. 2019; 01;85(2):214-8.

6. Haugen, B. R., Alexander, E. K., Bible, K. C., et al. 2015 American Thyroid Association Management Guidelines for Adult Patients with Thyroid Nodules and Differentiated Thyroid Cancer: The American Thyroid Association Guidelines Task Force on Thyroid Nodules and Differentiated Thyroid Cancer. Thyroid. 2016;26(1): 33-6.

7. Sulibhavi A, Rubin SJ, Park J, et al. Preventative and management strategies of hypocalcemia after thyroidectomy among surgeons: An international survey study. Am J Otolaryngol. 2020;41(3):102394.

8. Cusano NE and Bilezikian JP. Signs and Symptoms of Hypoparathyroidism. Endocrinol Metab Clin North Am. 2018;47(4): 759-70.

9. Dedivitis RA, Aires FT and Cernea CR. Hypoparathyroidism after thyroidectomy: prevention, assessment and management. Curr Opin Otolaryngol Head Neck Surg. 2017;25(2):142-6. 
10. Mazotas IG and Wang TS. The role and timing of parathyroid hormone determination after total thyroidectomy. Gland Surg. 2017;6:S3848.

11.Palmhag D, Brydolf J, Zedenius J, et al. A Single Parathyroid Hormone Measurement Two Hours after a Thyroidectomy Reliably Predicts Permanent Hypoparathyroidism. Scand J Surg. 2020; 9:1457496920913666.

12. Castro A, Del Rio L and Gavilan J. Stratifying the Risk of Developing Clinical Hypocalcemia after Thyroidectomy with Parathyroid Hormone. Otolaryngol Head Neck Surg. 2018;158(1):76-82.

13. Tay D., Cremers S., \& Bilezikian J. P. Optimal dosing and delivery of parathyroid hormone and its analogues for osteoporosis and hypoparathyroidism - translating the pharmacology. British Journal of Clinical Pharmacology. 2017;84(2): 252-67.
14. Algarni M, Alzahrani R, Dionigi G, et al. Parathyroid hormone and serum calcium levels measurements as predictors of postoperative hypocalcemia in total thyroidectomy. Gland Surg. 2017;6(5):428-32.

15. Chhetri $M$ and Bononi P. SUN-498 Normocalcemic Tetany: Is Parathyroid Insufficiency a Real Thing?. J Endocr Soc. 2019;3(Suppl 1):SUN-498.

16. Grigorovici A, Varcus F, Mogoș S, et al. Hypocalcemia After Thyroidectomy For Advanced Local Malignancies. Rev Chim (Bucharest) 2019; 70(3): 1053-7.

17. Lončar I, Dulfer RR, Massolt ET, et al. Postoperative parathyroid hormone levels as a predictor for persistent hypoparathyroidism. Eur J Endocrinol. 2020;183(2):149-59.

18. Orloff L. A., Wiseman S. M., Bernet V. J., et al. American Thyroid Association Statement on Postoperative Hypoparathyroidism: Diagnosis, Prevention, and Management in Adults. Thyroid. 2018;28(7): 830-41. 